\title{
Wideband Characterization of Power Amplifiers Using Undersampling
}

\author{
Per Niklas Landin ${ }^{1,2}$, Charles Nader ${ }^{1,2}$, Niclas Björsell' ${ }^{1}$, Magnus Isaksson ${ }^{1}$, David Wisell ${ }^{3}$, \\ Peter Händel $^{1,2}$, Olav Andersen ${ }^{4}$, and Niclas Keskitalo ${ }^{1,3}$ \\ ${ }^{1}$ Center for RF Measurement Technology, University of Gävle, Gävle, SE-801 76, Sweden \\ ${ }^{2}$ Signal Processing Lab, Royal Institute of Technology, Stockholm, SE-100 44, Sweden \\ ${ }^{3}$ Ericsson AB, Stockholm, SE-164 80, Sweden \\ ${ }^{4}$ Ericsson AB, Gävle, Box 6206, SE-800 06, Sweden
}

\begin{abstract}
In this paper a radio frequency power amplifier is measured and characterized by the use of undersampling based on the generalized Zhu-Frank sampling theorem. A test system has been designed allowing the bandwidth of the stimuli signal to be $100 \mathrm{MHz}$ in the characterization process. That would not be possible with any vector signal analyzer on the market. One of the more challenging problem within the proposed concept is the model validation process. Here, two different techniques for model validation are proposed, the multitone and the spectrum scan validation methods.
\end{abstract}

Index Terms - Communication system performance, modeling, nonlinear systems, power amplifiers, radio transmitters.

\section{INTRODUCTION}

Contemporary wide band code division multiple access (WCDMA) standards utilize bandwidth of $3.84 \mathrm{MHz}$. The 3GPP long term evolution (LTE) supports scalable bandwidths up to $20 \mathrm{MHz}$, and its continuation LTE advanced considerably more than that. Moreover, mobile worldwide interoperability for microwave access is supporting up to 28 $\mathrm{MHz}$. It is anticipated that future wireless communication systems will be more and more broad-band. The power amplifier (PA) is a key radio component in any wireless communication system, and behavioral modeling of its inputoutput characteristics a developed research area. It is also possible that signals from different bands and of different standards may share the same PA.

Considering the PA as a nonlinear dynamic device, the spectral support of its outputs does not only cover the spectral support of the input, but also the adjacent channels. Relying on the Nyquist-Shannon sampling theorem, sampling rates of several hundreds of $\mathrm{MHz}$ are required to sample the amplifier output according to the classical sampling theorem. For bandwidths at this order of magnitude, there are no available analog-digital converters (ADCs) with the required dynamic range, and thus there is a strong need for alternative radio frequency measurement technologies to circumvent this major obstacle. Alternatives include the use of time-interleaved ADCs, (frequency stitching employing repetitive amplifier input [1], or sub-sampling based on the seminal work by Zhu [2]. In short, Zhu's work implies that if there is a static invertable function that compresses the spectral support of an analog signal, it is sufficient to sample it with a speed corresponding to twice the bandwidth of the compressed signal. After ideal pulse-modulation to obtain the reconstructed signal, the inverse of the compressing function is applied to reconstructed data to obtain a full-band signal. The results of $\mathrm{Zhu}$ were later on generalized to nonlinear dynamic systems of certain classes by [3] and [4].

Motivated by the fundamental results on sampling theory for nonlinear system identification, under-sampling (in the Nyquist-Shannon context) has been identified as an emerging radio frequency measurement technology for wireless communication PAs, mostly in terms of theoretical studies. In the paper by Wisell [5], it was shown that by using real measurements on a 3G WCDMA PA employing different sampling rates spanning from some $4 \mathrm{MHz}$ up to $40 \mathrm{MHz}$, the amplifier model kept comparable performance using lower sampling rates. The work in [5] was extended in [6].

In the current work, we take the work of Wisell a step further by increasing the bandwidth of the signal to first 50 and then $96 \mathrm{MHz}$. This has been possible due to a specially designed test setup described in Section II [7]. The theory of the model identification is given in Section III and the results are presented in Section IV. Zhu-Frank sampling can utilize the whole Nyquist bandwidth of the ADC for model identification. However, model validation requires special solutions that are discussed in Section $\mathrm{V}$, followed by the conclusions in Section VI.

\section{TEST SETUP}

Zhu-Frank generalized sampling theorem (ZFGST) does not decrease the bandwidth required by the measurement system; only the sampling rate can be decreased. To utilize ZFGST one cannot rely on the vector signal analyzers on the market today since the intermediate frequency (IF) bandwidth preceding the ADC sampler front-end is limited to increase the dynamic range by avoiding noise folding from the broadband noise. Thus, a specially designed test system has been designed for PA characterization based on ZFGST.

In order to master the wide bandwidth requirements, the test-bed has an ultra wideband radio frequency (RF) front-end. The RF input frequency range is $500-2700 \mathrm{MHz}$ and the amplitude range is -10 to $+10 \mathrm{dBm}$ for dynamic range depending on the signal. The output amplifier has been designed with a frequency range of $20-1000 \mathrm{MHz}$ and $14 \mathrm{~dB}$ gain. In total this results in a front-end with exceptional 
properties. It has a $1000 \mathrm{MHz}$ bandwidth within $\pm 1.5 \mathrm{~dB}$ amplitude variations. It can handle up to $30 \mathrm{dBm}$ peak with close to $50 \mathrm{dBm}$ third interception point. That is well enough for the subsequent 12-bit pipelined ADC intended for direct IF sampling that operates up to 210 MSPS conversion rate with analog bandwidth of $700 \mathrm{MHz}$. A frame grabber interfaces to the ADC and in real-time records with a data length of 2 MSamples.

\section{MODEL IDENTIFICATION}

The model identification procedure has been described in [7]. Sampled input and output data records were measured at different time instants with the described measurement system. Synchronization of the acquired time series was needed before model identification.

Since the measurement system does not provide any possibilities of precise triggering (on the order of tenths of a sample interval) and the physical run-time through the system is unknown, the synchronization has been done using crosscorrelation and phase-compensation [8] to obtain sub-sample synchronization.

The synchronization was made in two steps. The first step was a rough synchronization on sample basis using crosscorrelation to find the "delay" between the measured input and output signals. The second step was a sub-sample synchronization to find the linear phase offset in the frequency domain.

The models were identified by minimizing the mean square error (MSE) of the measured output and the model output. As model structure, the commonly used parallel Hammerstein (PH) model [9] is chosen. The PH is defined by its nonlinear order $P$ and the memory length $\mathrm{M}$. Such a model is henceforth denoted $\operatorname{PH}(P, M)$. The models were identified using the measured quantities of the input and output signals, known at the specific time instant, and formed to a model-specific regression matrix $\Phi$. The non-linear model behavior is absorbed by $\Phi$. It was described with the model predictor

$$
\hat{y}(n)=\theta^{T} \Phi
$$

which is linear in the parameters $\theta$. The least-squares estimation problem is then addressed as an over determined set of equations, linear in the parameters. Powerful and simple methods can then be used when determining those parameters.

\section{EXPERIMENTAL AND RESULTS}

The tested amplifier is a LDMOS PA intended for being used in base stations in the $3^{\text {rd }}$ generation of mobile communications. It has $52 \mathrm{~dB}$ gain and a maximum rated input power of $1 \mathrm{dBm}$. This PA is designed for use in the 2110-2170 MHz band. In small-signal S21-measurements the frequency range with variations less than $0.5 \mathrm{~dB}$ is $2100-$ $2220 \mathrm{MHz}$; therefore the chosen center frequency is 2160 $\mathrm{MHz}$.
Due to the non-flat gain in the signal bandwidth, memory effects stronger than usual are expected. To obtain an accurate model these variations must be considered. The linear part of the PH model is simply a FIR-filter. With the variations within the signal bandwidth it is not necessarily true that the first coefficient of this filter is the largest coefficient. To check for possible "small" initial coefficients in the linear FIR-filter, a delay in the output signal was introduced with one sample at a time and identification was done for each such delay. The NMSE was then computed for comparison. In no case was more than 10 samples delay tested.

For the normal $3.84 \mathrm{MHz}$ WCDMA signal the model with lowest normalized MSE (NMSE) was the $\mathrm{PH}(9,4)$ with a NMSE of $-40.2 \mathrm{~dB}$ and an adjacent channel error power ratio (ACEPR) of $-56.5 \mathrm{~dB}$ [10]. No additional delays were required to obtain the latter model. These results with the found model order and model errors are in line with the results from [8] for this particular PA.

The model order with lowest NMSE for the $50 \mathrm{MHz}$ wide signal was the $\mathrm{PH}(9,7)$. In Fig. 1 the measured input, output and the model error for the $\mathrm{PH}(9,7)$ are shown. The necessary delay due to the wide bandwidth in this case was one sample yielding an improvement of $0.4 \mathrm{~dB}$ in NMSE as compared to using no extra delays.

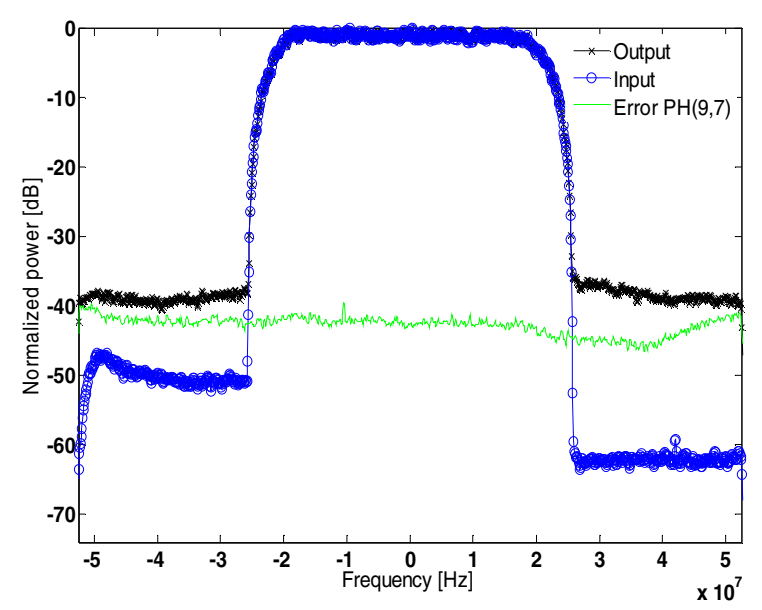

Fig. 1. The power spectra of the input, output and the model error of a $\mathrm{PH}(9,7)$ for the $50 \mathrm{MHz}$ input signal.

For the $96 \mathrm{MHz}$ wide signal the most suitable model based on NMSE was found to be a $\operatorname{PH}(9,9)$ with a NMSE of -32.8 $\mathrm{dB}$ and a delay of 3 samples compared to what the synchronization found. Adding this delay improved the NMSE by $0.5 \mathrm{~dB}$ compared to no delay and same model order.

\section{MODEL VALIDATION}

As shown in [6], [11]-[13] undersampling of the output signal of the PA can be used for the purpose of PA modeling with little or no loss in modeling performance. Common model performance evaluation criteria for PA behavioral 
models are NMSE, ACEPR and weighted error-to-spectral power ratio (WESPR). However, data sampled according to the ZFGST does only allow direct evaluation of NMSE. The NMSE has earlier [14]-[15] been shown to be an inadequate metric for model performance evaluation. In fact, ACEPR was in [16] found to be the best low-complexity metric to identify nonlinear mismatches.

The different frequency components of the output signal of the PA, due to aliasing, fall in the same frequency bins after the Zhu-Frank sampling making it impossible to separate linear from nonlinear model errors. In the following two alternative solutions are discussed.

\section{A. Multitone}

Here a multitone based approach to the model validation problem is proposed. Multitones have been used extensively for PA modeling purposes and their suitability for this task is well established in numerous papers e.g. [17] and the references therein. First, assume that a PA model has been extracted using Zhu-Frank sampling. The model can now be validated by using a multitone or a set of multitones according to [17] and Zhu-Frank sampling under the condition that the sampling frequency is set in such a manner that the intermodulation (IM) products generated in the PA, after the aliasing, fall on frequencies (with some margin) at which there is no input signal. This is in practice a mild requirement that still gives sufficient freedom to design the input signal and set the sampling frequency. The principle is illustrated in Fig. 2.

A complex four-tone signal at baseband with a normalized bandwidth of 60 is passed through a fifth order nonlinearity. The output signal then has a normalized bandwidth of 300 . The output signal is sampled using a sampling clock of 79 . This will cause the IM products to alias multiple times as shown and it is still possible to determine the amplitude and phase of them and, thus, to compute the frequency domain evaluation criteria ACEPR and WESPR.

The model validation is done by comparing the amplitudes and phases of the different IM products of the measured output of the PA and of the output from the model. Numerous techniques to swiftly determine the amplitude and phase of multitone signals of this kind exist. Here a method like the one presented in [18] is recommended. In this manner, it is possible to calculate the equivalent of adjacent channel leakage ratio [19] by adding the power of the IM products that fall in the adjacent channels. Further, for each IM product an error vector is calculated. The power of these error vectors can then be added and compared to the channel power, allowing the computation of a WESPR similar to ACEPR.

Using a fine frequency grid in the multitone signal makes the signal closely resemble a spectrum continuous signal and the calculated validation criteria close to the ones that would have been obtained with such a signal.

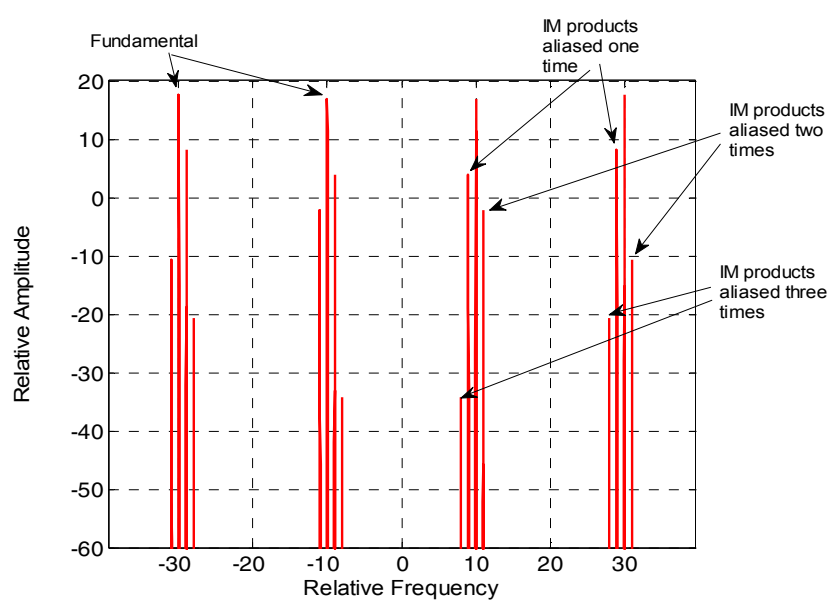

Fig. 2. Illustration of undersampling.

\section{B. Spectrum Scan}

The spectrum using Zhu-Frank sampling contains information from the true frequencies and the frequencies aliased back from higher Nyquist bands.

$$
\mathbf{A}_{\mathbf{d}}(\omega)=\sum_{k=0}^{\infty} \mathbf{I A}_{\mathbf{c}, 1}\left(k f_{s}\right)+\mathbf{Q} \mathbf{A}_{\mathbf{c}, 2}\left(\frac{f_{s}}{2}+k f_{s}\right)
$$

where $\mathbf{A}_{\mathbf{d}}=\left[\mathrm{A}_{\mathrm{d}}(0) \ldots \mathrm{A}_{\mathrm{d}}(\pi)\right]^{\mathrm{T}}$ is the sampled spectrum vector, $\mathbf{A}_{\mathbf{c}, 1}(f)$ is the IF spectrum for all frequencies within an odd Nyquist band starting with the frequency $f, \mathbf{A}_{\mathbf{c}, 2}(f)$ is the corresponding spectrum for even Nyquist bands, $f_{\mathrm{s}}$ is the sampling frequency, $\mathbf{I}$ is the unity matrix and $\mathbf{Q}$ is a matrix with zeros except on the sub-antidiagonal where it is -1 .

In practice, a low pass filter on the $\mathrm{ADC}$ input will remove frequencies outside the interesting frequency band and thus, in the following discussion an ideal low pass filter with a cut-off frequency at $K \cdot f_{s}$ will be considered. The IF spectrum, $\mathbf{A}_{\mathbf{C}}$, cannot be recovered directly from the sampled spectrum. However, by having a sequence of measurements, where the frequency of the local oscillator (LO), $f_{\mathrm{LO}}$, is changed between each measurements the complete spectrum can be recovered. In order to preserve information within a frequency bin the frequency step of the LO should be an integer, $s$, multiplied by the distance in frequency between two adjacent bins in the FFT. That is $f_{\mathrm{s}}$ divided by the data length, $N$. The notation in (2) will change to include different sets of measurements.

$$
\mathbf{A}_{\mathbf{d}}(\omega, l)=\sum_{k=0}^{K} \mathbf{I A}_{\mathbf{c}, 1}\left(k f_{s}+l s \frac{f_{s}}{N}\right)+\mathbf{Q A}_{\mathbf{c}, 2}\left(\frac{f_{s}}{2}+k f_{s}+l s \frac{f_{s}}{N}\right)(3)
$$

where $l$ is an index for the measurement series, $l=[0 \ldots L-1]$.

Several measurements will form the set of equations: 


$$
\left[\begin{array}{c}
A_{d}(\omega, 0) \\
\vdots \\
A_{d}(\omega, L-1)
\end{array}\right]=\left[\begin{array}{ccccc}
\mathbf{I} & \mathbf{Q} & \mathbf{I} & \cdots & \mathbf{0} \\
& \ddots & \ddots & \ddots & \\
\mathbf{0} & \cdots & \mathbf{I} & \mathbf{Q} & \mathbf{I}
\end{array}\right]\left[\begin{array}{c}
A_{c}\left(f_{0}\right) \\
\vdots \\
A_{c}\left(\frac{f_{s}}{2}+k f_{s}+(L-1) s \frac{f_{s}}{N}\right)
\end{array}\right]
$$

Under the right conditions, (4) can be used to recover the IF spectrum and to compute ACEPR and WESPR. The DC components should be excluded and the matrix must be quadratic and full rank.

The matrix can be quadratic by excluding the highest frequency components and full rank can be achieved by using proper step length, $s$. It can be shown that $s=N / 2-1$ will fulfil the requirement for full rank.

\section{CONCLUSIONS}

The ZFGST for the purpose of PA behavioral modeling was tested with different input signals of varying bandwidths, going from $3.84 \mathrm{MHz}$ through $50 \mathrm{MHz}$ to $96 \mathrm{MHz}$. Main difference in the models was the amount of required linear memory due to gain variations. For the wider signals, the normal cross-correlation based synchronization was no longer sufficient to find the optimal linear FIR-filter in the model. It was shown that introducing additional delays in the output signal as compared to the input signal improved the model performance with up to $0.5 \mathrm{~dB}$ for the same model order.

Validation of models extracted using the ZFGST was done using the NMSE. As has been shown in [10] and [14]-[15], the NMSE is not a well-suited criteria for PA behavioral model performance evaluation. However, due to the aliasing, criteria using out-of-band frequencies could not be used without special methods. In this paper two different methods are suggested. Multitone signals and frequency planning makes it possible to characterize IM products in-band and use criteria like WESPR. Another method is to achieve full-spectrum coverage by variation of the local oscillator frequency in the downconverter and thereby acquire signals for validation of ZFGST-extracted models.

\section{ACKNOWLEDGEMENT}

This work was supported by Ericsson AB, Freescale Semiconductor Nordic AB, Infineon Technologies Nordic AB, Knowledge Foundation, NOTE AB, Rohde\&Schwarz AB and Syntronic AB.

\section{REFERENCES}

[1] D. Wisell, D. Rönnow, and P. Händel, "A technique to extend the bandwidth of a power amplifier test-bed," IEEE Trans. Instrum. Meas., vol. 56, pp. 1488-1494, 2007.

[2] Y. M. Zhu, "Generalized sampling theorem," IEEE Trans. Circuits Syst., vol. 39, pp. 587-588, 1992.
[3] W. A. Frank, "Sampling requirements for Volterra system identification," IEEE, Signal Processing Lett., vol. 3, pp. 266268, 1996.

[4] J. Tsimbinos, and K. V. Lever, "Sampling frequency requirements for identification and compensation of nonlinear systems," in Acoustics, Speech, and Signal Processing. ICASSP94., IEEE, vol. 3, pp. 513-516, 1994.

[5] D. Wisell, "Exploring the sample rate limitation for modeling of power amplifiers," presented at IMEKO 2006 Conf. Dig., Rio de Janeiro, 2006.

[6] D. Wisell, and P. Händel, "Implementation considerations on the use of Zhu's general sampling theorem for characterization of power amplifiers," in Instrumentation and Measurement Technology Conf. Proc., 2007, pp. 1-4.

[7] O. Andersen, N. Björsell, and N. Keskitalo, "A test-bed designed to utilize Zhu's general sampling theorem to characterize power amplifiers," in $I^{2} M T C$ 2009, Singapore, 2007, to be published.

[8] M. Isaksson, D. Wisell, and D. Rönnow, "A comparative analysis of behavioral models for RF power amplifiers," IEEE Trans. Microwave Theory Tech., vol. 54, no. 1, pp. 348-359, Jan. 2006

[9] M.S. Heutmaker, E Wu, and J.R. Welch, "Envelope distortion models with memory improve the prediction of spectral regrowth for some RF amplifiers," in ARFTG Conference Digest-Fall, $48^{\text {th }}, 1996$, vol. 30, pp. 10-15.

[10] M. Isaksson, D. Wisell, and D. Rönnow, "Wideband dynamic modeling of power amplifiers using radial-basis function neural networks," IEEE Trans. Microwave Theory Tech., vol. 53, pp. 3422-3428, 2005.

[11] D. Wisell, "A baseband time domain measurement system for dynamic characterization of power amplifiers with high dynamic range over large bandwidths," in Instrumentation and Measurement Technology Conf., 2003. Proc. of the 20th IEEE, 2003, vol. 2, pp. 1177-1180.

[12] P. Singerl and H. Koeppl, "A low-rate identification method for digital predistorters based on Volterra kernel interpolation," in 48th Midwest Symposium on Circuits and Systems, 2005, vol. 2, pp.1533-1536.

[13] P. Singerl and H. Koeppl, "Volterra kernel interpolation for system modeling and predistortion purposes," in International Symposium on Signals, Circuits and Systems, 2005, vol. 1, pp. 251-254.

[14] P. Landin, M. Isaksson and P. Händel, "Comparison of evaluation criteria for power amplifier behavioral modeling", in IEEE MTT-S Int. Microwave Symp. Dig., Atlanta, GA, USA, 2008, pp. 1441-1444.

[15] D. Wisell, M. Isaksson and N. Keskitalo, "A general evaluation criteria for behavioral power amplifier modeling," in ARFTG 69, Honolulu, USA, 2007, pp. 251-255.

[16] D. Schreurs, M. O' Broma, A. A. Goacher and M. Gadringer, "RF Power Amplifier Behavioral Modeling," Cambridge University, Press 2009, 2008.

[17] N. B. Carvalho, K. A. Remley, D. Schreurs and K. C. Gard, "Multisine signals for wireless system test and design", Microwave Magazine, vol. 9, no. 3, pp. 122-138, Jun. 2008.

[18] D. Wisell, B. Rudlund, and D. Rönnow, "Characterization of memory effects in RF power amplifiers using digital two-tone measurements," IEEE Trans. Instrum. Meas., vol. 56, pp. 27572766, 2007.

[19] ETSI, “3GPP TS 25.141 V6.3.0.” 